\title{
Evidências de validade e consistência interna de tarefas de analogia gramatical
}

\author{
Márcia Maria Peruzzi Elia da Mota \\ Universidade do Estado do Rio de Janeiro \\ Universidade Salgado de Oliveira \\ Acácia Aparecida Angeli dos Santos \\ Universidade São Francisco \\ Silvia Brilhante Guimarães \\ Universidade do Estado do Rio de Janeiro
}

\section{Resumo}

O presente estudo investigou a validade de duas tarefas de analogia gramatical, focando nos morfemas derivacionais e flexionais. Participaram da pesquisa 217 crianças de escolas públicas matriculadas no segundo, terceiro e quarto ano do ensino fundamental. As crianças responderam a uma tarefa de consciência fonológica (RAFC) e duas de consciência morfológica. Foram encontradas evidências de validade de critério, pois os escores se diferenciaram pelos anos escolares para o escore composto e para a tarefa focando na morfologia derivacional. Outra evidência de validade foi detectada quando se verificou a associação dos escores do RACF com as tarefas de consciência morfológica. O escore composto da tarefa obteve índices de consistência interna adequados, mas com carência, Alfa de Cronbach de 0,66. Enfim, os resultados mostram que o escore composto da tarefa é sensível para mensurar a habilidade morfológica das crianças.

Palavras-chave: consciência metalinguística; habilidades linguísticas; consciência morfológica.

\begin{abstract}
Validity and internal consistency evidence for grammatical analogy task. The present study investigated the validity of two tasks of grammatical analogy test focusing in derivational and flexional morphology. Two-hundred and seventeen children drawn from public schools enrolled in the second, third and fourth year of elementary school participated in the research. The children answer a task of phonological awareness (RACF) and two of morphological awareness. Evidence of validity for discrimination between series was found for the composite score of morphological awareness and for the derivational morphology task. Evidence of validity for the morphological awareness composite task was also met when its association with the RACF. The internal consistency task felt short of criterion and was adequate, Cronbach's Alpha of 0.66 . The results show that the grammatical analogy task is a task that helps you access the morphological awareness of children.
\end{abstract}

Keywords: metalinguistic awareness; linguistic abilities; morphological awareness.

\section{Resumen}

Evidencias de validez y consistencia interna de tareas de analogía gramatical. Este estudio investigó la validez de dos tareas de analogía gramatical, centrándose en los morfemas derivativos y flexivos. 217 niños de escuelas públicas matriculadas en segundo, tercero y cuarto de primaria participaron del estudio. Los niños fueron sometidos a una tarea de conciencia fonológica (RACF) y dos más de conciencia morfológica. Se encontraron pruebas de validez de criterio, ya que las puntuaciones se diferenciaban según los cursos escolares para la puntuación compuesta y para la tarea en lo que a la morfología derivativa se refiere. Otra prueba de validez fue detectada al verificarse la asociación entre las puntuaciones RACF y las tareas de conciencia morfológica. La puntuación compuesta de la tarea obtuvo índices de consistencia interna adecuados, pero mostrando carencias. Cronbach Alfa de 0,66. En resumen, los resultados muestran que la puntuación compuesta de la tarea es sensible para medir la habilidad morfológica en los niños.

Palabras clave: conciencia metalinguística; habilidades lingüísticas; consciencia morfológica. 
C onsciência morfológica é uma habilidade metalinguística definida como a habilidade de refletir sobre os morfemas das palavras (Carlisle, 1995). A literatura especializada na área tem demonstrado a importância da consciência morfológica para aquisição da língua escrita em diferentes ortografias, tal como em português (Mota \& Silva, 2007, Mota, Annibal, \& Lima, 2008, Mota et al., 2012); em francês (Casalis \& Louis Alexandre, 2000; Colé, Marec-Breton, Royer, \& Gombert, 2003; Duncan, Casalis, \& Colé, 2009), em coreano (Wang, Ko, \& Choi, 2009) e em chinês (Liu \& Mcbride-Chang, 2010).

Há várias razões pelas quais a consciência morfológica é importante para leitura e escrita, pois ela auxilia a leitura à medida que ajuda a criança a reconhecer palavras que ainda não conhece ou que nunca encontrou em um texto. Palavras morfologicamente complexas podem ter seu significado compreendido se o leitor conhece a origem da palavra. Por exemplo, a palavra "hospitalizado" é mais facilmente compreendida se a criança conhece a palavra de origem "hospital". Além disso, os conhecimentos sintático-semânticos utilizados no processamento da consciência morfológica podem contribuir para a leitura, uma vez que na compreensão de um texto é necessário integrar esse tipo de informação (Tong, Deacon, Parrila, Cain, \& Kirby, 2011). Na escrita, a consciência morfológica provê suporte para que as crianças decidam sobre a ortografia de palavras ambíguas como "açucarado" que vem da palavra "açúcar". Importante salientar que os morfemas são as menores unidades em que podemos dividir as palavras, com significado próprio.

No português, Mota et al. (2008) mostraram que a consciência morfológica contribui para o processamento da leitura e escrita. As autoras utilizaram uma tarefa de analogia gramatical que foi inicialmente desenvolvida por Nunes, Bryant e Bindman (1997) e adaptada do inglês para o português por Mota (1996). Dessa forma, a versão utilizada por Mota et al. (2008) foi uma versão revisada, considerando a especificidade da morfologia do português.

A tarefa de analogia gramatical consiste em itens, nos quais a criança deve produzir uma palavra morfologicamente complexa a partir de uma palavra-alvo, aplicando a mesma relação de derivação de um par previamente dado. Por exemplo, para o par "pedra-pedreiro"; a criança deve inferir a relação morfológica e produzir uma nova palavra de forma a manter a mesma relação do par anterior para o novo par "leite-___?".

Não há uma forma única de se avaliar a consciência morfológica. De fato, dentro do escopo das habilidades metalinguísticas há diferentes tarefas que focalizam diferentes unidades linguísticas e exigem diferentes níveis de processamento cognitivo e metalinguístico (Mota, 2012b). Por um lado, há tarefas de consciência morfológica que envolvem apenas o julgamento sobre a estrutura morfológica das palavras, como a tarefa de decisão lexical usada por Mota et al. (2012). Neste caso a criança tem que decidir se as palavras vêm de uma mesma família ou não. Por exemplo, "chique e chiqueiro são da mesma família?”. As tarefas de julgamento exigem um grau de habilidade metalinguística mais implícita, visto que não envolvem a manipulação intencional da estrutura das palavras. Por outro lado, existem tarefas de analogia gramatical cognitivamente mais complexa que avaliam a habilidade da criança em manipular morfemas, por exemplo, ao pedir para criança analisar o par "leite-leiteiro" e completar "pão-__?" ela terá que entender a relação morfológica sobre o par e generalizá-la para outras situações, exigindo um grau razoável de domínio das habilidades metalinguísticas (Deacon \& Kirby, 2004).

Neste estudo escolhemos investigar as características psicométricas de uma tarefa de analogia gramatical que envolve a manipulação de morfemas, pois ela tem sido muito comumente associada ao desempenho na leitura no português (Mota et al., 2008). Consideramos que é necessário que se estabeleçam mais evidências sobre se ela mede realmente o que se propõe a medir, de forma a evitar que interpretações equivocadas sejam feitas.

Além da questão da demanda cognitiva da tarefa, uma questão de destaque quando investigamos as habilidades metalinguísticas é o foco das unidades linguísticas que envolvem nossas investigações (Mota, 2012a). No caso da consciência morfológica, uma primeira grande divisão que deve ser considerada é entre dois grupos de morfemas que parecem ser processados de formas diferentes pelas crianças: morfemas derivacionais e flexionais.

Um estudo que demostrou essas diferenças de processamento foi realizado por Carlisle (1995). A autora deu a crianças de educação infantil e primeiro ano uma tarefa de produção morfológica. Nesta tarefa a criança tinha que completar uma frase com uma palavra faltando, por exemplo, à criança era dada uma palavra alvo como "fazenda", e ela tinha que completar uma frase como "Meu tio é um __?". Um terço das palavras omitidas eram flexões, por exemplo, "Tenho um copo. Maria tem dois __?". Outro terço eram palavras derivadas com uma relação fonologicamente transparente (a derivação segue o mesmo princípio fonológico) com a palavra alvo ("fazenda-fazendeiro"), e último terço eram de palavras derivadas, mas com uma relação opaca (a derivação não segue o mesmo princípio fonológico) com a palavra alvo ("razão-racional"). Os resultados mostraram que nas três situações, era mais fácil para a criança completar as frases que requeriam as flexões do que as que requeriam derivações. As crianças da educação infantil tiveram mais dificuldades em resolver esta tarefa. $O$ índice de acerto foi de $1,9 \%$ para as palavras derivadas com relações opacas, $22,8 \%$ para as palavras derivadas com relação transparente e $36,5 \%$ para as palavras flexionadas. Estes resultados indicam também que o processamento das flexões é mais fácil para as crianças do que o processamento das derivações.

Outro estudo sobre esse tema foi realizado por Deacon e Bryant (2005). Esses pesquisadores deram a crianças de 5 a 8 anos de idade um teste de escrita, no qual as crianças tinham que escrever palavras morfologicamente simples (com um morfema) e palavras morfologicamente complexas (com dois morfemas). Metade das palavras de dois morfemas eram palavras derivadas e a outra metade eram palavras flexionadas. As palavras tinham 
o mesmo som final, de modo que as dificuldades encontradas não poderiam ser atribuídas a diferenças na ortografia ou ao som das palavras. Por exemplo, a palavra notion (com um morfema) e a palavra connection (com dois morfemas) terminavam com o mesmo som. Os autores predisseram que se as crianças processam a morfologia da língua, elas teriam uma facilidade maior em escrever o som final das palavras quando eles eram morfemas, do que quando não eram. No caso das palavras morfologicamente complexas, esses sons finais eram unidades destacáveis das palavras, nas simples, eles compunham a palavra como um todo.

Os resultados confirmaram a hipótese de trabalho assumida por Deacon e Bryant (2005), mostrando que as crianças escreviam mais corretamente os sons finais das palavras quando eram morfemas do que quando não eram. Porém, a análise do tipo de morfema escrito mostrou que este resultado era referente apenas às flexões. Os autores concluíram que a facilidade encontrada na escrita dos morfemas flexionais possivelmente ocorria porque na morfologia derivacional há uma mudança na classe gramatical das palavras morfologicamente complexas, o que não ocorre com a morfologia flexional. Assim, seria mais fácil para as crianças entender as relações morfêmicas nas flexões do que nas derivações. Esses resultados mostram que esses tipos de morfemas têm características diversas e, embora possamos formar um conjunto de tarefas de consciência morfológica, elas precisarão ser balanceadas em relação aos tipos de morfema focalizado. Dessa forma, poderão ser evitados vieses nos resultados de aferição dessa habilidade.

Tem havido um aumento na preocupação em se criar novas tarefas com evidências de validades estabelecidas que avaliem a consciência morfológica. Vianna (2012) teve dificuldades em discriminar adolescentes quanto ao desempenho em tarefas de consciência morfológica por falta de estudos de critérios de validade e de instrumentos que abarquem diferentes faixas etárias. No caso do estudo do autor, a dificuldade foi com tarefas que discriminassem a consciência morfológica nos anos finais do Ensino Fundamental. Este problema não tem sido abordado na literatura internacional, que em um primeiro momento se preocupou prioritariamente com questões conceituais em relação aos tipos de morfemas que estariam em foco e definições do que poderíamos considerar consciência morfológica em oposição à consciência sintática ou às diferenças entre processamento morfológico e conhecimento morfológico em relação à consciência sintática (Correa, 2004; 2009).

Assim, precisamos nos dedicar à pesquisa das qualidades psicométricas de instrumentos de consciência morfológica com o intuito de verificar se eles medem aquilo a que se propõe a medir. Os psicometristas têm fortes argumentos a favor da importância das qualidades psicométricas das medidas usadas nos diferentes contextos, incluindo o educacional (Nunes \& Primi, 2010; Urbina, 2007).

Se a consciência morfológica é necessária para a aquisição da escrita, tarefas que avaliam essa habilidade são de grande importância educacional e clínica. Foi para responder essa lacuna na literatura que essa pesquisa foi delineada, pressupondo que esta é uma questão relevante. Acredita-se que o avanço do conhecimento sobre essas questões poderá auxiliar na identificação de crianças com dificuldades nesta área e fornecer elementos para a elaboração de programas de intervenções que favoreçam o desenvolvimento da consciência morfológica em crianças que mostrem dificuldades nessas tarefas.

Dada a importância do tema para a alfabetização, o acesso a tarefas bem construídas com evidências de validade estabelecidas é importante para profissionais que trabalhem com esta temática, visto que os resultados obtidos poderão possibilitar inferências mais adequadas para as interpretações feitas. Sob essa perspectiva, o objetivo do presente estudo foi investigar evidências de validade de um teste de Analogia Gramatical dividido em duas partes: Analogia derivacional e Analogia flexional.

Essa tarefa foi inicialmente criada no inglês por Nunes et al. (1997). Escolhemos investigá-la pois é a que melhor tem se correlacionado com a leitura e escrita no português (Mota, 1996; Mota, Annibal, \& Lima, 2008). Além disso, Tong et al. (2011) demonstraram que o processamento da morfologia derivacional e flexional podem contribuir de forma diferente, para leitura e escrita, ao longo do desenvolvimento escolar. Assim, essa é uma tarefa versátil no que diz respeito à avaliação da consciência morfológica dos indivíduos. Vale destacar que serão investigadas evidências de validade de critério (ano escolar) e evidências oriundas da análise da relação com outras variáveis, aqui especificamente da relação com medida que avalia construto similar (processamento fonológico). Serão também estimados os índices de consistência interna das tarefas.

\section{Método}

\section{Participantes}

Os participantes da pesquisa foram 134 crianças, ambos os sexos, entre 6 e 13 anos do segundo ao quarto ano do ensino fundamental, regularmente matriculadas em quatro escolas públicas de uma cidade do interior do estado de Minas Gerais. A amostra foi de conveniência, sendo que entre os alunos de $2^{\circ}$ ano $(n=30)$ a média de idade foi de 7,43 anos $(D P=$ $0,63)$, entre os de $3^{\circ}$ ano $(n=52)$ a média foi de 8,31 anos (DP $=0,47)$ e entre os de $4^{\circ}$ ano $(n=52)$ a média de idade foi de 9,27 anos $(D P=0,49)$. A amostra foi não aleatória e o critério de inclusão na pesquisa foi a assinatura dos responsáveis de um termo de consentimento livre e esclarecido, tal como submetido ao Comitê de Ética e Pesquisa. Além da concordância dos pais, as crianças também foram elucidadas sobre a participação voluntária na pesquisa.

\section{Instrumentos}

Analogia Gramatical (adaptado de Nunes, Bryant, \& Bindman, 1997). A tarefa de analogia gramatical foi inicialmente adaptada por Mota (1996). A autora, no entanto, só focou no desenvolvimento de itens de analogia flexional. O desenvolvimento de estudos recentes que chamam a atenção da morfologia derivacional 
para o desenvolvimento da leitura escrita chamou atenção para necessidade da ampliação da tarefa para focar nesse aspecto do processamento morfológico também.

Morfologia Derivacional. A tarefa analisa a capacidade da criança em manejar os morfemas. A tarefa foi adaptada, considerando-se a especificidade da morfologia derivacional da língua portuguesa. Nesse sentido, foram organizados inicialmente 10 itens, reduzindo-se depois para oito para melhorar os índices de consistência interna. A decisão por seis itens também se deveu à ideia de se manter para os dois números de morfemas um mesmo número de itens, facilitando a criação de um conjunto de tarefas de consciência morfológica. Em cada um, a criança tem que ser capaz de formar uma palavra morfologicamente complexa a partir de uma palavra alvo. Por exemplo, "tênis-tenista; arte-___?". Os critérios estabelecidos para correção preveem a atribuição de um ponto para cada acerto e de zero ponto para cada erro.

Morfologia Flexional. A tarefa adaptada requer da criança a capacidade de identificar a modificação morfossintática num par de palavras e depois aplicá-la em outro par, de forma semelhante à tarefa envolvendo a morfologia derivacional. Essa tarefa foi reduzida de 12 para oito itens para melhorar o nível de consistência interna, dois se referindo à concordância verbal, quatro de flexão de número e dois de gênero. "Leitor-leu; escritor-___?" para morfologia derivacional e para morfologia flexional "Anda-andou; olha-___?". Para cada item correto, foi dado um ponto e, para cada erro, zero pontos.

Roteiro de avaliação da consciência fonológica - RACF (Santos, 1996). A tarefa verifica a dificuldade da criança em identificar o fonema inicial, final e o do meio das palavras. Os itens são organizados em três conjuntos, cada um com três itens de prática e cinco de teste, perfazendo um total de 15 itens. No primeiro conjunto, a criança é solicitada a identificar, entre três palavras diferentes (palavras-teste), qual começa com o mesmo som que uma palavra-estímulo. Por exemplo, o som inicial de "copo" (palavra-estímulo) entre as palavras-teste "cobra - sapo - lata". O mesmo procedimento acontece com os outros conjuntos, só que com os sons finais e os do meio, respectivamente.

O RACF tem evidência de validade experimental demonstrada no estudo de Santos (1996), no qual foram comparadas crianças de educação infantil antes e depois de um treino para desenvolvimento da consciência fonológica. Os resultados mostraram que o instrumento é suficientemente sensível para mostrar os efeitos desse treinamento, visto que os sujeitos do grupo experimental obtiveram escores significativamente superiores aos dos sujeitos do grupo de controle. Em estudo posterior foram estabelecidas evidências de validade de critério, na qual se identificou sua sensibilidade para separar o desempenho das crianças por ano escolar (Suehiro \& Santos, 2011).

\section{Procedimentos}

O presente estudo integra um projeto de pesquisa mais amplo denominado "Avaliação Psicométrica de medidas de consciência metalinguística", sendo aqui descritos os procedimentos para as tarefas contempladas neste artigo. Respeitou-se os procedimentos éticos para estudos com seres humanos estabelecidos pela Resolução CNS n 466/12, bem como as demais orientações do Comitê de Ética em Pesquisa.

Para a coleta de dados, as aplicações das tarefas e testes selecionados ocorreram em locais reservados nas próprias escolas. Para aplicação do RACF mostrou-se para a criança uma palavra modelo e depois outras três, para que ela indicasse, respectivamente em cada um dos conjuntos de itens, quais das três começavam/terminavam ou tinham o som do meio igual ao do som da palavra modelo. Nesta mesma sessão aplicou-se a tarefa de consciência morfológica, de analogia gramatical derivacional apresentando à criança um par de palavras relacionadas entre si, depois ela ouviu mais uma palavra, que ela deveria completar com outra. "Por exemplo, pedra-pedreiro, leite-__?" e assim, sucessivamente, com os outros pares de palavras. Na sessão seguinte aplicou-se a tarefa de analogia gramatical - morfologia flexional, seguindo o mesmo procedimento utilizado para a derivacional. O tempo de duração das sessões foi de 20 a 30 minutos aproximadamente.

\section{Análise de dados}

Foram usadas provas da estatística não paramétrica para a análise de dados, considerando-se que a distribuição dos escores das variáveis dependentes não foi normal. Dessa forma, recorreu-se à prova de Kruskal-Wallis para comparar as diferenças dos escores entre os anos escolares, bem como às provas de correlações bisseriais de Spearman para explorar os dados obtidos.

\section{Resultados}

Com vistas ao estabelecimento da evidência de validade de critério relativa ao ano escolar, iniciou-se a comparação entre os escores obtidos em cada um deles. A Tabela 1 mostra os resultados das médias e desvios padrões para cada teste.

Tabela 1

Média da Idade e Desvio Padrão por Anos Escolar da Amostra para as Tarefas de Analogia Gramatical Rerivacional e Flexional

\begin{tabular}{cccc}
\hline Ano & Derivacional & Flexional & Morfologia total \\
& Média (dp) & Média (dp) & Média (dp) \\
\hline $2^{0}$ & $2,96(1,76)$ & $5,07(1,41)$ & $8,00(2,68)$ \\
$3^{0}$ & $3,92(1,44)$ & $5,60(0,63)$ & $9,51(1,64)$ \\
$4^{0}$ & $4,23(1,48)$ & $5,65(0,91)$ & $9,88(1,78)$ \\
\hline
\end{tabular}

Os resultados das análises Kruskal-Wallis que compararam os escores na tarefa de morfologia derivacional foram significativos para as diferenças entre os três anos escolares $(p=0,009)$. No entanto, para morfologia flexional o resultado não foi significativo $(p=0,09)$. Para o conjunto das tarefas, considerando o escore geral resultante da aplicação dos 12 itens, o resultado da diferença entre os anos escolares foi significativo $(p=0,003)$. 
Análises post hoc foram feitas, usando comparações com teste Mann Whitney para o escore composto, havendo diferenças entre o segundo e terceiro ano $(p=0,018)$ e para a morfologia derivacional $(p=0,015)$. Para o segundo e quarto ano as diferenças foram significativas para ambos os testes ao nível de significância $(p=0,01)$. Não houve diferenças entre o terceiro e quarto anos indicando que o terceiro ano parece ser uma etapa de transição no desenvolvimento da consciência morfológica.

Buscando evidências de validade entre medidas que avaliam construtos relacionados, os escores do RAFC, que é uma tarefa de consciência metalinguística (consciência fonológica), foram incluídos na análise de correlação não paramétrica realizada com os escores obtidos com as tarefas de consciência morfológica. O escore total no RACF correlacionou-se de forma moderada e estatisticamente significativa com a tarefa de analogia derivacional ( $R h o=0,42 ; p<0,001)$. Também a correlação entre o escore total no RACF e o escore obtido no escore na tarefa da analogia flexional mostrou correlações significativas, porém de magnitude fraca ( $R h o=0,30 ; p<0,001$ ). O escore geral, composto com todos os itens escore total no RACF correlacionou-se de forma moderada e estatisticamente significativa com o escore geral das tarefas de analogia derivacional ( $R h o=0,44 ; p<0,001)$. Os resultados estão apresentados na Tabela 2.

Tabela 2

Correlações de Spearman entre as Medidas de Habilidades Metalinguísticas

\begin{tabular}{cccc}
\hline & Flexional & Morfologia & RACF \\
& & total & total \\
\hline Derivacional & $0,26^{* *}$ & $0,93^{* *}$ & $0,42^{* *}$ \\
Flexional & & $0,55^{* *}$ & $0,30^{* *}$ \\
Morfologia total & & & $0,44^{* *}$ \\
\hline
\end{tabular}

$* * p<0,001$

A análise de consistência interna da tarefa de analogia focada na morfologia derivacional apresentou, após ajustes, com a redução de itens, índices moderados com carência, com alfa de Cronbach referente a 0,59. No caso da morfologia flexional os resultados foram semelhantes, o resultado final resultou numa tarefa com análise de consistência interna apresentando índices adequados, mas também com carência, com alfa de Cronbach de 0,61. O conjunto total de tarefas com 12 itens obteve índices aceitáveis de consistência interna (alfa de Cronbach $=0,66)$.

\section{Discussão}

De um modo geral, tem se apontado para importância de pesquisas que buscam estabelecer a validade de instrumentos de avaliação, graças à demonstração de que o uso de medidas para avaliação dos fenômenos deve ser baseado em evidências sustentadas por dados empíricos obtidos com estudos metodologicamente adequados (Nunes \& Primi, 2010; Urbina, 2007). Na área de habilidades metalinguísticas estudos apontam a importância de cuidar dos instrumentos utilizados para avaliar essa habilidade em crianças e adolescentes, que sejam não só teoricamente consistentes, como também que tenham características psicométricas adequadas (Mota, 2012, Santos \& Cunha, 2012; Viana, 2012).

Os dados do presente trabalho contribuíram para preencher uma lacuna na literatura no que diz respeito às tarefas de consciência morfológica e o estudo das características psicométricas das mesmas. Propôs-se uma tarefa composta de 12 itens, que acessa tanto aspectos específicos do conhecimento da morfologia derivacional como da flexional da criança.

Iniciou-se por tentar construir um instrumento que tivesse índices adequados de consistência interna. A tarefa com escore composto resultado das análises dos itens, foi formada, conforme exposto acima, por 12 itens e aproximou-se muito do índice consistência interna recomendada na área de psicometria (índice de consistência interna de 0,70). Esta tarefa de escore composto foi formada por seis itens que investigou a reflexão das crianças sobre aspectos da morfologia derivacional e seis da morfologia flexional. Para a tarefa de morfologia derivacional, os índices foram adequados com carência, enquanto que a tarefa de morfologia flexional, por outro lado, apresentou índices de consistência abaixo do desejável.

A morfologia flexional no português é complexa, engloba gênero, número e tempo dos verbos, podemos pensar que há diferentes níveis de processamento desses morfemas, e atingir a consistência interna com uma variedade grande de morfemas pode ficar difícil. Esta é uma questão que deve ser considerada se essa tarefa for modificada. Considerando-se que a estimativa de precisão pode variar segundo o contexto de investigação (DeVellis, 1991), a aceitação do padrão de 0,60 no presente estudo, nos leva a recomendar que os dados sejam interpretados com precaução. Estudos futuros deverão investigar alternativas para superar esta deficiência, sendo o aumento de itens uma das possibilidades a ser explorada.

Em relação às evidências de validade da tarefa de analogia gramatical, é importante ressaltar que ela discrimina entre os anos escolares. O resultado da análise que comparava nas tarefas do escore composto foi significativo para ano escolar e para os escores no teste de analogia gramatical focado nas derivações. A análise das médias do desempenho em morfologia flexional indica que as crianças dos três anos escolares tiveram efeitos próximos ao teto, mostrando que não houve discriminação entre essas etapas e que essa tarefa foi fácil para as crianças. A literatura demonstra que a morfologia derivacional é adquirida mais tarde do que a flexional (Deacon \& Bryant, 2005; Vianna, 2012). Logo, nossos resultados são consistentes com o da literatura da área.

Encontramos também evidência de validade para a tarefa de analogia gramatical quando comparada ao RACF. Embora, os resultados das correlações tenham sido fracos, o RACF correlacionou-se de forma estatisticamente significativa com os escores na tarefa de analogia gramatical com os morfemas 
flexionais e de forma moderada com os escores de analogia gramatical com morfemas derivacionais e na tarefa composta. $\mathrm{Na}$ introdução desse trabalho, argumentamos que o RAFC é uma medida validada de consciência metalinguística (Santos, 1996; Suehiro \& Santos, 2011). Dessa maneira, essas correlações apresentam suporte adicional para o estabelecimento de evidências de validade das tarefas de consciência morfológica.

Os resultados aqui apresentados mostram que a tarefa de analogia gramatical apresenta evidência de validade para avaliar a habilidade morfológica de crianças de segundo ao quarto ano do ensino fundamental. As análises do escore composto (derivacional e flexional) mostraram diferenças entre o segundo e terceiro ano o que não ocorreu para morfologia flexional. Para o segundo e quarto ano as diferenças foram significativas para ambas as tarefas. Não houve diferenças entre o terceiro e quarto anos indicando que o terceiro ano é o que merece mais investigação, haja vista o fato de que essa pode ser uma etapa escolar de transição no desenvolvimento da consciência morfológica. As crianças do terceiro ano tiveram um desempenho superior às do segundo ano, mas inferior às do quarto ano. Este em si é um resultado interessante sobre a sensibilidade das medidas. O fato de elas separarem os escores dos alunos da amostra por ano escolar confere evidência de validade de critério para aferir o construto da consciência morfológica.

Também os índices de correlação positiva dos escores obtidos entre os escores das medidas de consciência morfológica com o RACF, indicam que ambas estão medindo construtos similares e que elas compartilham uma parte da variância explicada do fenômeno. As correlações foram significativas para as três versões da tarefa: as duas de morfologia calculadas separadamente e àquela com escore geral.

A habilidade metalinguística é necessária para a aquisição da escrita como apontam vários estudos estrangeiros e nacionais (Carlisle, 1995; Deacon, Benere, \& Pasquarella, 2013; Duncan et al., 2009; Kruk \& Bergman, 2013; Miranda \& Mota, 2013; Mota et al, 2012). Sob essa perspectiva, os resultados do presente estudo foram tratados com o propósito de buscar evidências de validade de um instrumento de consciência morfológica que poderia servir como medida a ser utilizada em pesquisas no contexto educacional.

Recomenda-se cautela no seu uso como instrumento de rastreio na identificação de dificuldades de aprendizagem de leitura. Antes disso, as tarefas precisam ter suas características psicométricas aprimoradas, especialmente no que tange aos itens referentes à morfologia flexional. Acreditamos que, com base nos resultados encontrados, devem ser planejados novos estudos visando à criação de um conjunto de tarefas mais completo, que deverá ser aplicado em amostras mais amplas e diversificadas para que, posteriormente, possam ser usadas como ferramentas de trabalho, tanto nas práticas escolares no cotidiano da sala de aula, como na prática clínica.

\section{Referências}

Carlisle, J. F. (1995). Morphological awareness and early reading achievement. In L. B. Feldman (Org.), Morphological aspects of language processing (pp. 189-209). Hillsdale, NJ: Erlbaum.

Casalis, S., \& Louis-Alexandre, M. (2000). Morphological analysis, phonological analysis and learning to read French: A longitudinal study. Reading and Writing, 12, 303-335. doi: 10.1023/A:1008177205648

Colé, P., Marec-Breton, N., Royer, C., \& Gombert, J. (2003). Morphologie des mots et apprentissage de la lecture. Reeducation Orthophonic, 213, 57-60.

Conselho Nacional de Saúde (2012). Resolução CNS no 466/12, de 12 de dezembro de 2012. Recuperado de http://www.jusbrasil.com.br/diarios/55483111/ dou-secao-1-13-06-2013-pg-59

Correa, J. (2004). A avaliação da consciência sintática na criança: Uma análise metodológica. Psicologia, Teoria e Pesquisa, 20(1), 69-75. doi: 10.1590/ S0102-37722004000100009

Correa, J. (2009). Habilidades metalinguísticas relacionadas à sintaxe e a morfologia. In M. M. P. Mota (Org.), Desenvolvimento metalinguístico: Questões contemporâneas (pp. 55-76). São Paulo: Casa do Psicólogo.

Deacon, D., Benere, J., \& Pasquarella, A. (2013). Reciprocal relationship: Children's morphological awareness and their reading accuracy across grades 2 to 3. Developmental Psychology, 49(6), 1113-1126. doi: 10.1037/ a0029474

Deacon, S., \& Bryant, P. (2005). What young children do and do not know about the spelling of inflections and derivations. Developmental Science, 8(6), 583-594. doi: 10.1111/j.1467-7687.2005.00449.x

Deacon, S., \& Kirby, J. (2004). Morphological awareness: Just "more phonological"? The roles of morphological and phonological awareness in reading development. Applied Psycholinguistics, 25(2), 223-238. doi: 10.1017/S0142716404001110

DeVellis, R. F. (1991). Scale development: Theory and applications. Newbury Park, CA: SAGE Publications.

Duncan, L., Casalis, S., \& Colé, P. (2009). Early metalinguistic awareness of derivational morphology: Observations from comparison of English and French. Applied Psycholinguistics, 30(3), 405-440. doi: 10.1017/ S0142716409090213

Kruk, R., \& Bergman, K. (2013). The reciprocal relations between morphological processes and reading. Journal of Experimental Child Psychology, 114(1), 10-34. doi: 10.1016/j.jecp.2012.09.014

Liu, P. D., \& Mcbride-Chang, C. (2010). What is morphological awareness? Taping lexical compounding awareness in Chinese third graders. Journal of Educational Psychology, 102(1), 62-73. doi: 10.1037/a0016933

Miranda, L., \& Mota, M. M. P. E. (2013). Há uma relação específica entre consciência morfológica e reconhecimento de palavras? Psico-USF, 18(2), 241-247. doi: 10.1590/S1413-82712013000200008

Mota, M. M. P. E. (1996). The role of grammatical knowledge in spelling (Tese de doutorado não publicada). Oxford University.

Mota, M. M. P. E. (2012a). Considerações metodológicas e conceituais sobre a construção de instrumentos de avaliação das habilidades metalinguísticas. Avaliação Psicológica 11(1), 77-82. Recuperado de http://pepsic.bvsalud. org/scielo.php?script=sci_arttext\&pid=S1677-04712012000100008\&ln $\mathrm{g}=\mathrm{pt} \& \ln \mathrm{ln}=\mathrm{pt}$

Mota, M. M. P. E. (2012b). Explorando a relação entre a consciência morfológica, processamento cognitivo e o desempenho na escrita. Estudos de PsicologiaCampinas, 29(1), 97-102. doi: 10.1590/S0103-166X2012000100010

Mota, M. M. P. E., \& Silva, K. (2007). Consciência morfológica e desenvolvimento ortográfico: Um estudo exploratório. Psicologia em Pesquisa, 1(2), 86-92. Recuperado de http://pepsic.bvsalud.org/scielo. php?script=sci_arttext\&pid=S1982-12472007000200009\&lng=pt\&tlng $=p t$ 
Mota, M. M. P. E., Annibal, L., \& Lima, S. (2008). A morfologia derivacional contribui para a leitura e escrita no português? Psicologia Reflexão e Crítica, 21(2), 311-318. doi: 10.1590/S0102-79722008000200017

Mota, M. M. P. E., E., Lisboa, R., Dias, J., Gontijo, R., Paiva, N., Mansur-Lisboa, S., ... Santos, A. A. A. (2009). Relação entre consciência morfológica e leitura contextual medida pelo teste de Cloze. Psicologia: Reflexão e Crítica, 22(2), 223-229. doi: 10.1590/S0102-79722009000200008

Mota, M. M. P. E., Xavier, L., Almeida, W., Gammom, C., Ferreira, T. O., Honorato, J., ... Silva, R. (2012). Desenvolvimento da morfologia derivacional nos primeiros anos do ensino fundamental. Psicologia em Pesquisa, 6(1), 13-18. Recuperado de http://www.uff.br/psicologiaempesquisa/files/2012/09/ v6n1a03.pdf

Nunes, C. H. S., \& Primi, R. (2010). Aspectos técnicos e conceituais da ficha de avaliação dos testes psicológicos. In Conselho Federal de Psicologia - CFP (Org.), Avaliação psicológica: Diretrizes na regulamentação da profissão (pp. 101-128). Brasília: CFP.

Nunes, T,. Bryant, P., \& Bindman, M. (1997). Morphological spelling strategies: Developmental stages and processes. Developmental Psychology, 33(4), 637-649. doi: 10.1037/0012-1649.33.4.637

Santos, A. A. A. (1996). A influência da consciência fonológica na aquisição da leitura e da escrita. In F. F. Sisto, G. Oliveira, L. Fini, M. T. Souza, \& R. Brenelli (Orgs.), Atuação psicopedagógica e aprendizagem escolar (pp. 213247). Petrópolis: Vozes.

Santos, A. A. A., \& Cunha, N. B. (2012). Consciência metatextual: Evidências de validade para instrumento de medida. Psico-USF, 17(2), 233-241. doi: 10.1590/S1413-82712012000200007

Suehiro, A. C. B., \& Santos, A. A. A. (2011). Roteiro de avaliação da consciência fonológica (RACF). Acta Colombiana de Psicologia, 14, 147-154. Recuperado de http://www.scielo.org.co/scielo.php?script=sci_arttext\&pid=S012391552011000100013\&lng=en\&tlng=es. .

Tong, X., Deacon, S., Parrila, S., Cain, K., \& Kirby, J. (2011). Morphological awareness: A key to understanding poor reading comprehension in English. Journal of Educational Psychological Association, 103(3), 523-534. doi: $10.1037 \% 2 \mathrm{Fa} 0023495$

Urbina, S. (2007). Fundamentos da testagem psicológica. Porto Alegre: Artmed.

Viana, P. (2012). Leitura e desenvolvimento da consciência morfológica em adolescente do ensino fundamental (Dissertação de Mestrado não publicada). Universidade Salgado de Oliveira.

Wang. M., Ko, I. Y., \& Choi, J. (2009). The importance of morphological awareness in Korean-English. Contemporary Educational Psychology, 34, 132-142. doi: 10.1016/j.cedpsych.2008.12.002 
Márcia Maria Peruzzi Elia da Mota, Doutora em Psicologia pela Universidade de Oxford - Inglaterra, Pósdoutora em Avaliação Psicológica pela Universidade São Francisco (USF), é Vice-diretora do Instituto de Psicologia da Universidade do Estado do Rio de Janeiro (UERJ) e Professora Titular do Programa de Pós-Graduação em Psicologia da Universidade Salgado de Oliveira (UNIVERSO). Endereço para correspondência: Rua Marechal Deodoro 217, $2^{\circ}$ andar, Centro, Niterói, CEP: 24030-060. E-mail: mmotapsi@gmail.com

Acácia Aparecida Angeli dos Santos, Doutora em Psicologia Escolar e do Desenvolvimento Humano pela Universidade de São Paulo (USP), é Professora Titular da Universidade São Francisco (USF). E-mail: acacia.angeli@gmail.com

Silvia Brilhante Guimarães, Mestre em Psicologia pela Universidade Federal de Juiz de Fora, é Doutoranda em Psicologia pela Universidade do Estado do Rio de Janeiro (UERJ). E-mail:

silvia_brilhante@yahoo.com.br 